\title{
Análisis de la variabilidad del peso de los ladrillos y la importancia de estos parámetros para la producción en la ladrillera Las Casitas, Valledupar-Cesar
}

Luis Quintero' ${ }^{1}$ 0. Cantillo², Dilan Robles ${ }^{3}$

\section{Resumen}

El principal motivo de nuestra investigación es la necesidad de una mejor organización a las entidades mineras del departamento del Cesar, nos enfocamos en la concepción de Las Casitas a través de un análisis detallado del peso de los ladrillos y la resistencia que tienen al ser sometidos a un proceso de compresión, empezando por un diagnóstico general para determinar las falencias, virtudes y características del proceso de elaboración del ladrillo. Debido a las falencias presentadas en la homogeneidad del producto decidimos llevar a cabo esta investigación. Fue importante para nosotros estudiar sobre los aspectos técnicos en relación con la producción.

Palabras claves: alfareros, azadones, compresión, concesión, deformación, explotación, hornos, Pit, resistencia.

\footnotetext{
IIngeniero agroindustrial, docente del programa Ingeniería Geológica de la Fundación Universitaria del Área Andina sede Valledupar, Colombia. Correo: lquintero34dareandina.edu.co

2Estudiantes de Ingeniería Geológica de la Fundación Universitaria del Área Andina sede Valledupar, Colombia. Correo: ocantillo3ßa estudiantes.areandina.edu.co

Estudiantes de Ingeniería Geológica de la Fundación Universitaria del Área Andina sede Valledupar, Colombia. Correo: drobles6la estudiantes.areandina.edu.co
} 


\section{Introducción}

La producción de ladrillo artesanal en la ladrillera Las Casitas, municipio de Valledupar, es de muy bajo nivel técnico, sin ningún tipo de maquinaria, ni estandarización de procesos, ni registros de ningún tipo. Por lo tanto, el producto no es normalizado y esto conlleva a que la producción no tenga la calidad ideal. Esto es un problema para la industria de la construcción, ya que no hay claridad en las características técnicas y especificaciones del ladrillo y puede influir en la calidad de las edificaciones.

Teniendo en cuenta lo anterior, se plantea el uso de un método sencillo que nos ayude a deducir que tan homogéneo sale el producto final, teniendo en cuenta que la elaboración del producto es totalmente artesanal y sin ningún nivel tecnológico. El peso de los ladrillos como indicador de la homogeneidad del producto puede mostrar que las unidades no son homogéneas, dando diferencias grandes en el peso de los ladrillos entre el mismo horno y entre varios hornos dentro de la ladrillera Las Casitas, este método es rápido, las mediciones del peso de los ladrillos son tomadas en campo de manera ágil y con una precisión de $0,01 \mathrm{~kg}$, que ha sido suficiente para definir las variaciones del peso.
Los ladrillos deben presentar variaciones grandes en el peso entre una unidad productiva y otra dentro de la misma ladrillera debido a que la técnica de fabricación en totalmente manual, sin procedimientos de control de la homogeneidad del producto.

Todo producto industrial requiere de una estandarización, para tener un producto de calidad que garantice al cliente su máximo aprovechamiento y le dé garantías de que todas las unidades producidas tengan las mismas características o especificaciones técnicas. Esto es importante porque la industria artesanal debe competir con industrias de bloques mecanizadas, frente a las que se ve en desventaja por la calidad y mala estandarización de su producto.

Con esta investigación se inicia la caracterización de los ladrillos producidos artesanalmente, que puede aportar luces sobre la futura estandarización de los procesos para su elaboración.

Se puede encontrar en los datos recolectados que el proceso de elaboración del ladrillo utilizado en Las Casitas es de manera artesanal, es decir, que se realiza de manera empírica, según el cocimiento de los alfareros. Además, se comprobó que la resistencia de los la- 
drillos ante el esfuerzo producido por la compresión no supera los $5 \mathrm{Mpa}$. Según lo planteado por la NTC 4205 (Norma Técnica Colombiana, 2011), se debe considerar como el defecto principal el no cumplimiento de la resistencia. Se concluyó que en esta cantera no tienen ningún fundamento teórico para elaborar el ladrillo de manera óptima; por tal razón, los ladrillos no cumplen con los parámetros de resistencia establecidos en la NSR98 (Norma Sismo Resistente Colombiana, 1998).

\section{Materiales y métodos}

Nos apoyamos en los reglamentos de elaboración propuestos por Gonzáles (2008) para organizar la información, en la que se muestran los pasos del proceso de elaboración de manera detallada.

El sistema de explotación de la concesión es a cielo abierto y el método empleado es mediante banco único descendentes con alturas de, aproximadamente, uno a dos metros, aunque por su desorganización se pudieron ver bancos aproximadamente de cinco a diez metros, en los cuales se evidenció que no llevan una secuencia lógica para la extracción de material.

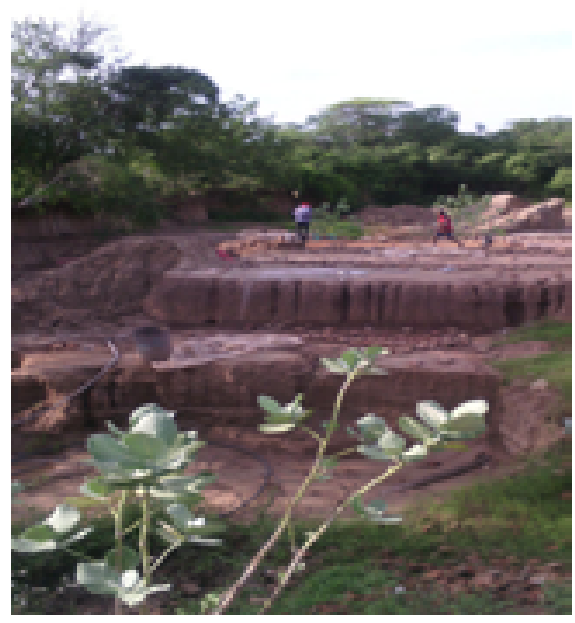

Figura 1. Frente de explotación de mina Las Casitas.

Fuente: elaboración propia.

Este arranque del material es realizado manualmente, proceso para el cual se utilizan azadones y palas; al material extraído se le vierte agua y con la pala se voltea, a la vez que se le añade el cisco de arroz o de arena, aditivo que le brinda mayor consistencia a la mezcla y ayuda a que el ladrillo no se fracture durante las etapas de secado y cocción. Para la maduración de la mezcla se deja reposar por 24 horas, con el agua suficiente y la cantidad necesaria que se tenga establecida. 


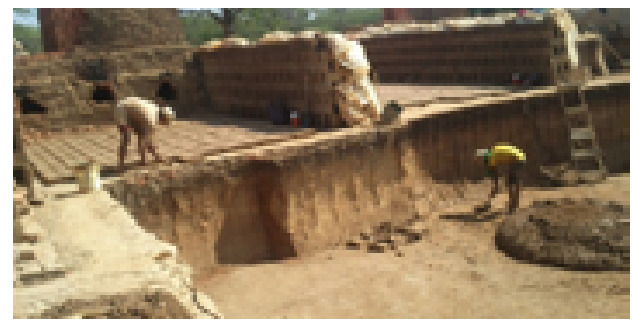

Figura 2. Extracción manual de arcilla Fuente: elacoración propia.

Este moldeo se realiza manualmente y consiste en rellenar con la mezcla del material arcilloso un espacio delimitado por un rectángulo de madera (molde), compactándola con las manos para que tome la forma con las dimensiones siguientes: longitud $28 \mathrm{~cm}$, profundidad $14 \mathrm{~cm}$, alto $8 \mathrm{~cm}$. Estos moldes son lavados con agua, para que el ladrillo terminado deslice fácilmente y no se adhiera a la madera. Esta actividad se lleva a cabo en los patios, en los que se forman hileras que cubren la superficie establecida para tal fin; allí duran 24 horas hasta que se compacta la mezcla; luego, se alisa (raspar con un cuchillo las superficies irregulares del ladrillo).

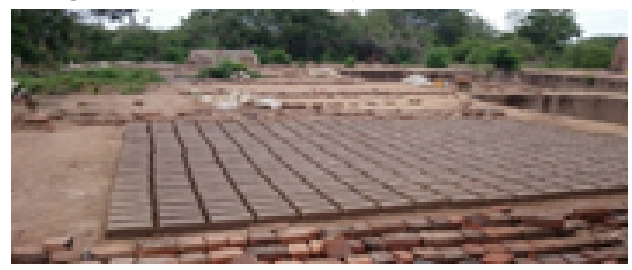

Figura 3. Moldeo para la elaboración del ladrillo

Fuente: elaboración propia.
El secado es realizado al aire libre, el cual demora dependiendo de la época; un tiempo de diez días para invierno y cinco días para verano. Los ladrillos van uno encima de otro, formando hileras, ubicados en forma transversal la hilera de arriba con respecto a la de abajo, alcanzando una altura aproximada de 1,2 metros, para que no haya fractura de los ladrillos en la parte inferior.

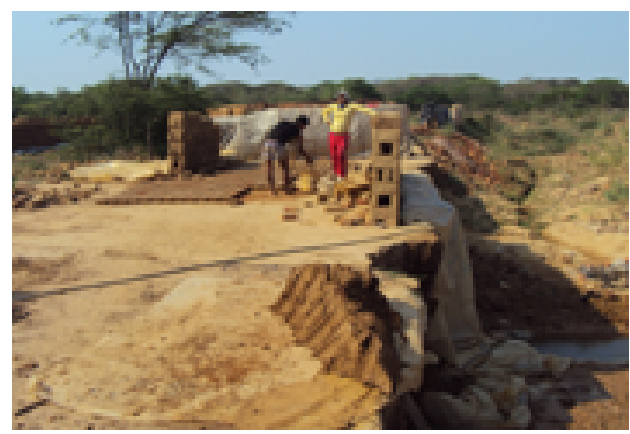

Figura 4. Secado del ladrillo

Fuente: elaboración propia.

La cocción de los ladrillos en el área de explotación se lleva a cabo en los hornos tipo pampa y se utiliza como combustible generalmente la leña; la frecuencia de quema varía en función de las condiciones meteorológicas y necesidades económicas del explotador.

La cocción de los ladrillos se produce en, aproximadamente, 48 horas. Cada horno tiene dimensiones variables. El combustible es suministrado por los carros cargadores de ladrillos que ha- 
cen cambio de materiales (ladrillos por leñas) con los dueños de los diferentes hornos.

Para mantener homogéneo el suministro de combustible se requiere alimentar por ambos lados de la siguiente manera: se enciende de un lado, este se encuentra destapado mientras que el lado opuesto se encuentra sellado con ladrillos y mezcla de material arcilloso; cuando se observa que la candela llega a la parte superior del horno, se sella de este lado y se descubre el otro; el tiempo que tarda este proceso es lo que se conoce como tiempo de quema. El tiempo de quema demora aproximadamente 36 horas.

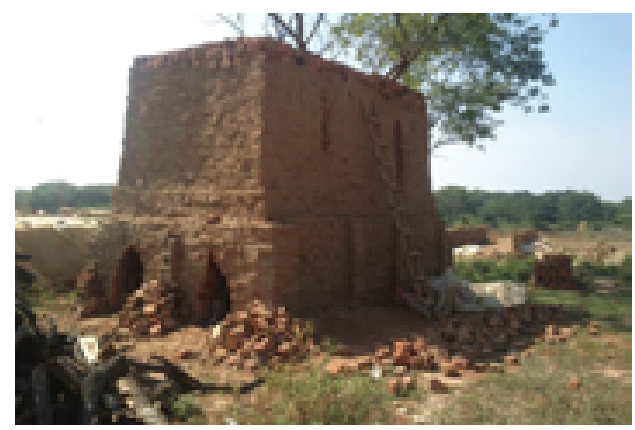

Figura 5. Horno tipo pampa para La cocción del ladrillo

Fuente: elaboración propia.

Una vez cocido y reposado el ladrillo se desarma los hornos, esta actividad es realizada por dos o tres personas en promedio, quienes con ayuda de una escalera inician retirando la tapa del horno, posteriormente quitan la camisa del horno que se refiere a toda la estructura del ladrillo del horno que anteriormente fue sellado con arcilla. Luego apilan los ladrillos cocinados para su posterior entrega.

También se establece el número total de unidades cocinadas y de unidades perdidas por mala cocción o preparación.

Una vez clasificado los ladrillos tienen dos destinos:

Los ladrillos son apilados y organizados a la espera de un comprador.

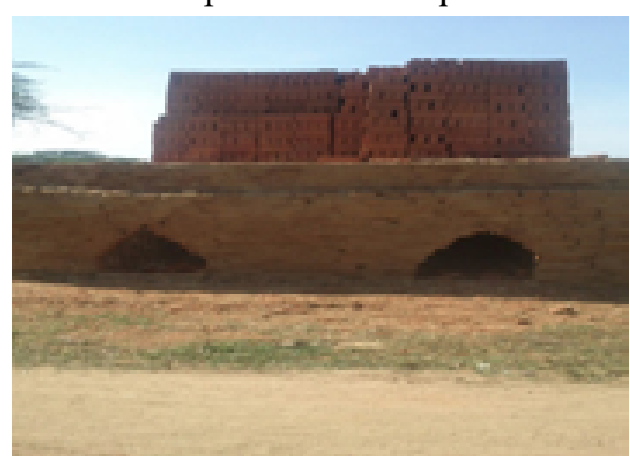

Figura 6. Clasificación de ladrillos Fuente: fotografía del autor.

- Los ladrillos que se han vendido antes de la cocción son cargados directamente del horno al vehículo que los transporta. 


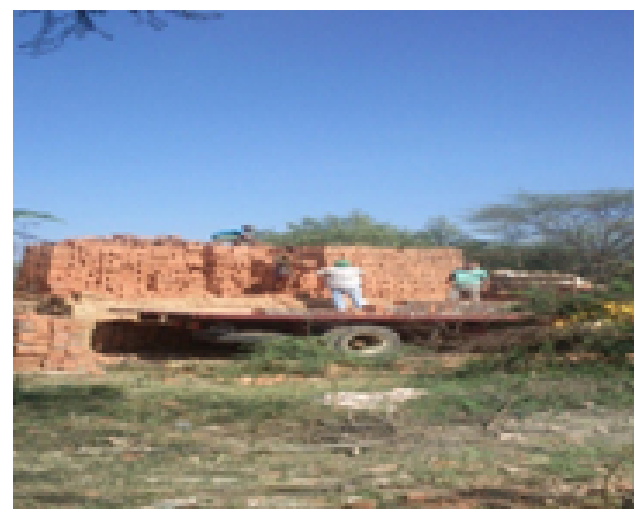

Figura 7. Cargue de ladrillos Fuente: elaboración propia.

La producción mensual es de 67500 ladrillos, que corresponde a la población total (Fuente: Junta de Acción Comunal Las Casitas, 2006).

Se midió el peso de diez ladrillos en los seis hornos más representativos, para un total de sesenta ladrillos como nuestra representativa.

\section{Tipos de muestreo}

Se plantean dos técnicas de trabajo: 1.Un análisis cuantitativo por muestreo estadístico aleatorio estratificado del peso de los ladrillos en diferentes unidades y sectores de la ladrillera.
2.Recolección de datos a través de cuestionario, que consiste en un conjunto de preguntas respecto de una o más variables, en este caso realizar el análisis de la percepción de los trabajadores sobre el parámetro del peso y su influencia en el proceso de fabricación de los ladrillos.

\section{Muestreo aleatorio estratificado del ladrillo}

Se divide la población en grupos en función de un carácter determinado y después se muestrea cada grupo aleatoriamente para obtener la parte proporcional de la muestra. Este método se aplica para evitar que por azar algún grupo de individuos esté menos representado que los otros.

Como primera técnica de muestreo se dividió la población de manera estratificada según el área en dos sectores, y dentro de ellos se escogieron seis hornos representativos para escoger la muestra aleatoriamente en cada uno de estos hornos, aproximadamente diez ladrillos por horno. 


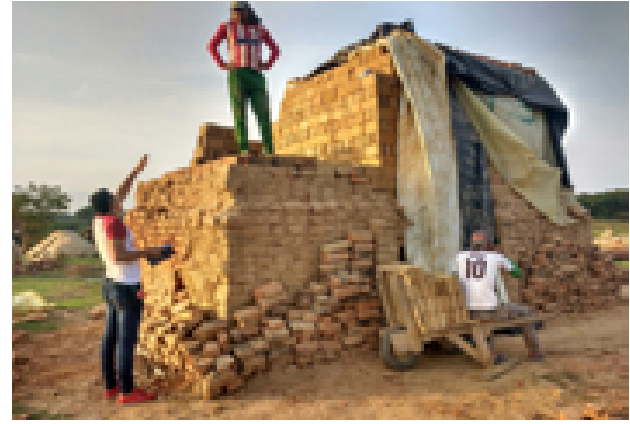

Figura 8. Horno en el que se ubican 10000 ladrillos de los cuales se escogieron $10 \mathrm{de}$ manera aleatoria Fuente: elaboración propia.

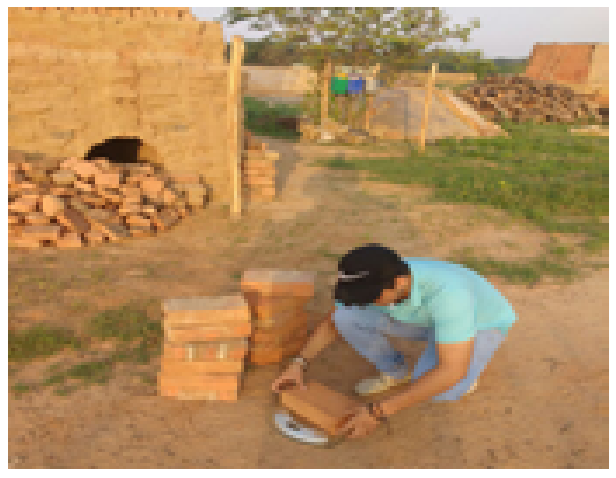

Figura 9. Proceso de pesaje de la muestra aleatoria tomada en el horno escogido estratificadamente Fuente: elaboración propia.

Para la medición del peso de los ladrillos se utilizó una balanza digital comercial, marca Kenwell, fabricada en acero inoxidable, vidrio templado y plástico, con las siguientes características técnicas:
- Capacidad: $150 \mathrm{~kg}$

- División: 100 gr

- Mecanismo: electrónico

- Material: vidrio templado y acero

- Plataforma de vidrio templado de $6 \mathrm{~mm}$

- Pantalla LCD de 52 x $24 \mathrm{~mm}$

- Encendido/apagado automático

- Funciona con pila 3V CR2032

- Indicador de sobrecargaIndicador de batería baja". (Biomed. com)

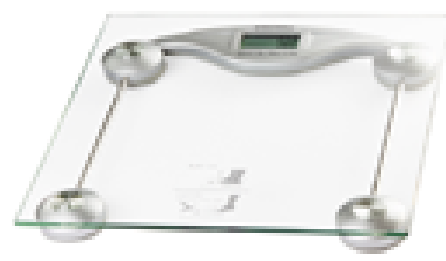

Figura 10. Instrumento de medición: Báscula. Fuente: fotografía tomada de página web de Badecol.

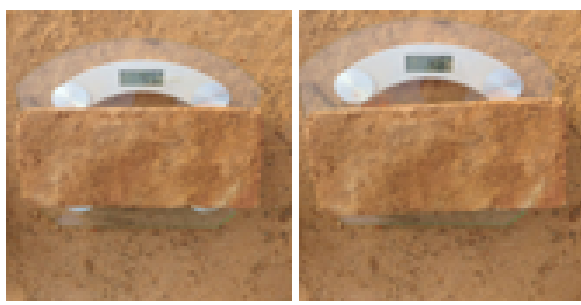

Figuras 11 y 12. Proceso de pesaje de las muestras.

Fuente: elaboración propia. 

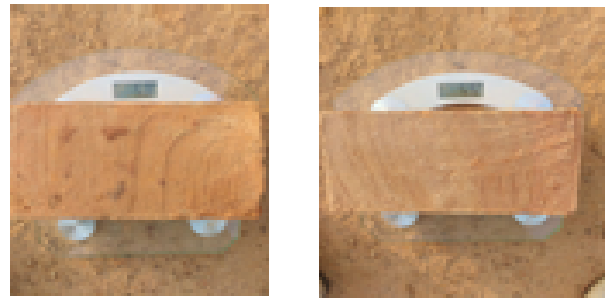

Figuras 13 y 14. Proceso de pesaje de las muestras.

Fuente: elaboración propia.

\section{Recolección de datos a través de cuestionario}

Consiste en un conjunto de preguntas respecto de una o más variables, en este caso realizar el análisis de la percepción de los trabajadores sobre el parámetro del peso y su influencia en el proceso de fabricación de los ladrillos.

Se realizó un cuestionario de nueve preguntas abiertas, diseñadas estratégicamente; siguiendo las indicaciones de Corral (2010), se ubicaron las preguntas de la siguiente manera:

Información de identificación

Pregunta 1. ¿Cómo se llama el dueño del horno?

\section{Información básica}

Pregunta 2. ¿Cuántos trabajadores hay en el horno? El objetivo de esta pregunta es definir el tamaño de empleados en la unidad productiva.
Pregunta 3. ¿Cuál es la producción por quema de ladrillo? El objetivo era conocer la producción real de la unidad productiva.

Pregunta 4. ¿En la última quema cuantos ladrillos hicieron? El objetivo era conocer la producción más cercana en el momento de tomar la muestra.

Pregunta 5. ¿Qué tipo de molde utilizó para el ladrillo? El objetivo era conocer las características físicas técnicas para la elaboración del ladrillo.

Pregunta 6. ¿Conoce usted cuánto pesa el ladrillo? El objetivo era conocer la percepción del peso del ladrillo que tiene el fabricante.

Pregunta 7. ¿Todos los ladrillos salen con la misma característica o varían? El objetivo era saber si el fabricante tenía idea de la estandarización de las características del ladrillo.

Pregunta 8. ¿Usted cree que el peso del ladrillo es importante para su producto? El objetivo era conocer la percepción del fabricante acerca de la importancia del peso del ladrillo.

Pregunta 9. ¿Cuántos ladrillos se dañan por quema? El objetivo era saber cuántos ladrillos se dañan o son desperdiciados en el proceso de su elaboración. 
El tipo de cuestionario utilizado fue un cuestionario oral o verbal a través de la entrevista personal o cara a cara, en la que nosotros como entrevistadores aplicamos el instrumento y registramos las respuestas.

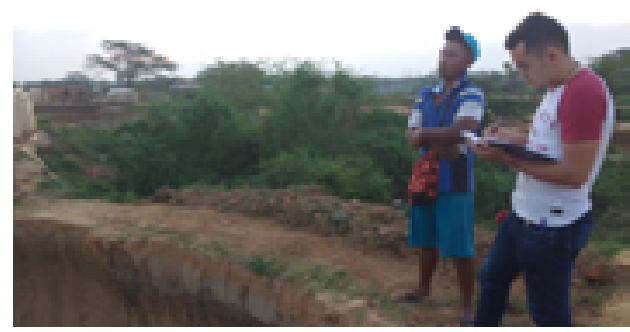

Figura 15. Recolección de datos a través de cuestionario

Fuente: elaboración propia.

\section{Resultados}

Una vez tomados los datos en campo, se digitaron en Microsoft Excel, generando la siguiente tabla organizada por el número de horno, los datos de peso están expresados en kilogramos.

Tabla 1. Datos del muestreo

\begin{tabular}{|c|c|c|c|c|c|}
\hline Fane I & 16anes? & mans 1 & Mosmed & Mans & atesese \\
\hline $45 \times$ & 35.4 & 678 & $3.1 \varepsilon_{4}$ & 44 & $53 \%$ \\
\hline 9004 & 8.18 & $.00 \%$ & $\pm x \in$ & 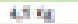 & $5.0 \times 4$ \\
\hline $53 \times 4$ & $32 \varepsilon_{4}$ & 484 & $x+\varepsilon_{4}$ & 4.4 & $5.5 \%$ \\
\hline ares & s.tes & $40 \mathrm{sg}$ & 2.36 & 436 & soxt \\
\hline $4.1 \times 4$ & $30 \varepsilon_{4}$ & $66 \%$ & $3.1 \varepsilon_{4}$ & 48 & $5.6 \mathrm{~kg}$ \\
\hline 4.84 & $4,2 x_{x}$ & $4 \pi$ & $\pm x \varepsilon_{y}$ & 46 & $5,2 \% 4$ \\
\hline 468 & 1.48 & $47 \pi$ & 34.6 & 45 & 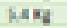 \\
\hline 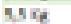 & 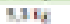 & 408 & MAE & ves & $100 \%$ \\
\hline $5.1 \times 4$ & $34 \varepsilon_{z}$ & UNg & 276 & 5.46 & $56 \mathrm{~kg}$ \\
\hline $9=8$ & s48 & $0 \%$ & \pm 18 & $48 \%$ & $5 \times 4$ \\
\hline
\end{tabular}

Fuente: elaboración propia
A los datos anteriores se les calculó el promedio del peso de los ladrillos por cada horno, la desviación estándar y la varianza, mostrados en la siguiente tabla:

Tabla 2. Análisis estadísticos de los datos

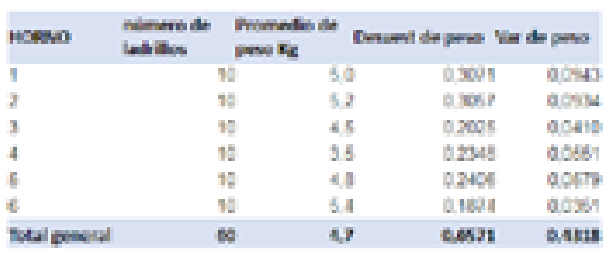

Fuente: elaboración propia.

El promedio general del peso de todos los ladrillos es de $4,7 \mathrm{~kg}$, sin embargo, existen variaciones significativas del peso promedio en los seis hornos. Se resalta que el horno cuatro tiene un promedio de $3,5 \mathrm{~kg}$ como valor mínimo promedio y el horno seis tiene un promedio de 5,4 $\mathrm{kg}$ como valor máximo promedio. Notamos, entonces una diferencia de casi $2 \mathrm{~kg}$ entre estos dos hornos, que corrobora la hipótesis de trabajo en la cual planteamos que el peso presenta variaciones grandes (ver tabla 2). 


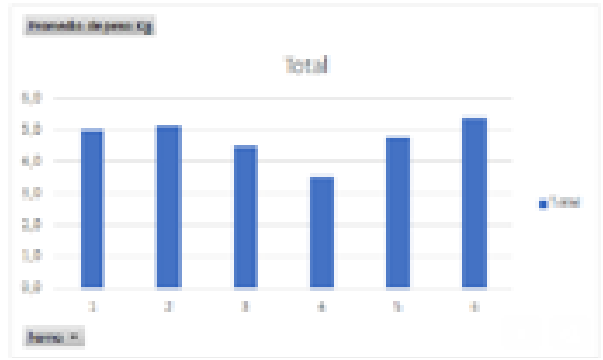

Figura 16. Gráfico de barras del promedio de peso para cada uno de los seis hornos Fuente: elaboración propia

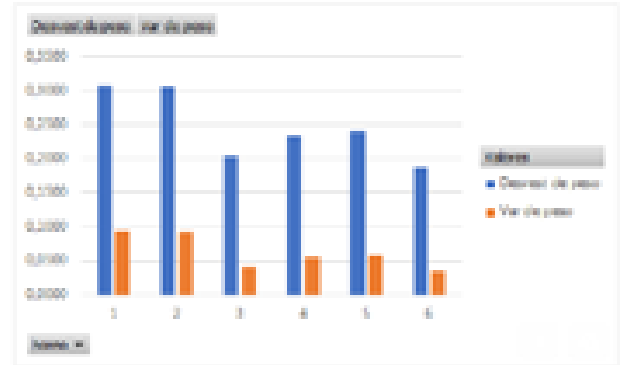

Figura 17. Gráfico de barras de la desviación estándar y varianza

Fuente: elaboración propia

En cuanto a los datos de desviación y variación estándar, nos arroja que el horno seis presenta los valores más bajos, esto nos quiere decir que los ladrillos de esta unidad no presentan tanta variabilidad como las otras unidades. Los hornos uno y dos presentan más variabilidad (ver figura 10).

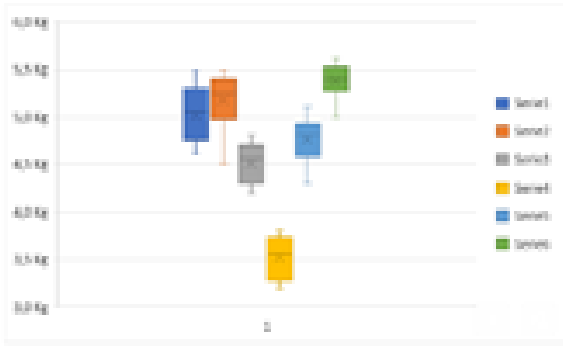

Figura 18. Gráfico de cajas y bigotes de los datos estadísticos Fuente: elaboración propia

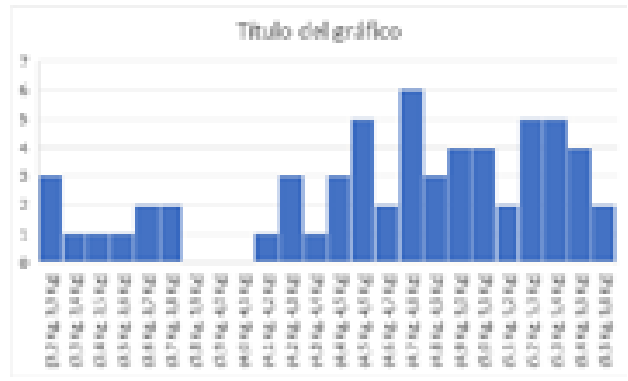

Figura19. Distribución normal de los datos Fuente: elaboración propia

Después de realizar el proceso de muestreo y de los pesos de los ladrillos, sometimos los ladrillos promedios a una prueba de compresión para medir su resistencia y obtuvimos los siguientes datos: 
Tabla 3. Datos del proceso de resistencia

\begin{tabular}{r|r|r|r|r|}
\hline peso & $\mathrm{kN}$ & area $\mathrm{cm} 2$ & $\mathrm{~Pa}$ *10Exp4 & Mpa \\
\hline 4873 & 154 & 397,6 & 387,32394 & 3,8732394 \\
\hline 5066 & 72,7 & 375,38 & 193,67041 & 1,9367041 \\
\hline 5027 & 166 & 368,28 & 450,744 & 4,50744 \\
\hline 5457 & 66,5 & 382,26 & 179,96536 & 1,7396536 \\
\hline 5304 & 113,16 & 356,4 & 317,50842 & 3,1750842 \\
\hline 4732 & 101,18 & 353,6 & 286,14253 & 2,8614253 \\
\hline 4804 & 104,47 & 372,6 & 280,38111 & 2,8038111 \\
\hline 4935 & 106,16 & 382,2 & 277,76033 & 2,7776033 \\
\hline
\end{tabular}

Fuente: elaboración propia

Tabla 4. Datos de la desviación estándar y varianza

compresion peso

\begin{tabular}{|l|r|r|}
\hline desv estandal & 0,9164056 & 248,43611 \\
\hline varianza & 0,8397993 & 61720,5 \\
\hline promedio & 2,9593701 & 5024,75 \\
\hline
\end{tabular}

Fuente: elaboración propia

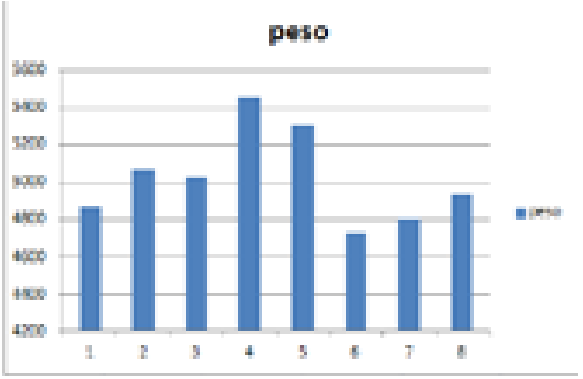

Figura 20. Distribución del peso en el proceso de resistencia

Fuente: elaboración propia

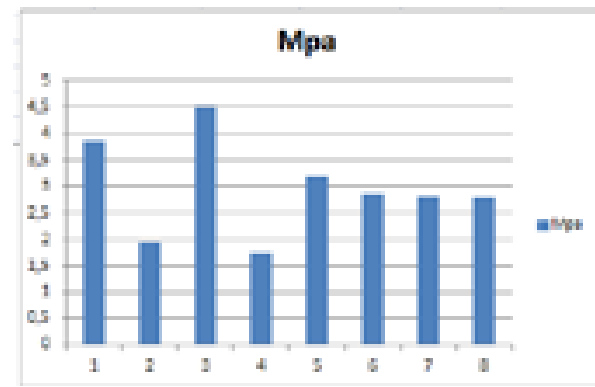

Figura 21. Distribución de resistencias Fuente: elaboración propia

Una vez obtuvimos los datos de la compresión, concluimos que el peso de los ladrillos no influye directamente con la resistencia de los ladrillos, deducimos que la resistencia de las unidades se ve afectada por otros factores, como la compactación de la mezcla y alguna alteración la elaboración normal de los ladrillos. 
Los resultados del cuestionario para cada una de las preguntas son los siguientes:

Pregunta 1. ¿Cómo se llama el dueño del horno?

Respuestas

Horno 1: Armando Salinas

Horno 2: Francisco Rincón

Horno 3: Heriberto Hinojosa

Horno 4: Omar Campo

Horno 5: Claudio Martínez

Horno 6: José Carrillo

Pregunta 2. ¿Cuántos trabajadores hay en el horno?

Respuestas

Horno 1: dos trabajadores

Horno 2: dos trabajadores

Horno 3: un trabajador

Horno 4: tres trabajadores

Horno 5: dos trabajadores

Horno 6: dos trabajadores

Pregunta 3. ¿Cuál es la producción por quema de ladrillo?

Respuestas
Horno 1: 11000 unidades

Horno 2: 10000 unidades

Horno 3: 10000 unidades

Horno 4: 13500 unidades

Horno 5: 10000 unidades

Horno 6: 13000 unidades

Pregunta 4. ¿En la última quema cuantos ladrillos hicieron?

Respuestas

Horno 1: se hicieron 12000 ladrillos

Horno 2: se hicieron 9000 ladrillos

Horno 3: se hicieron 9500 ladrillos

Horno 4: se hicieron 13000 ladrillos

Horno 5: se hicieron 10500 ladrillos

Horno 6: se hicieron 13500 ladrillos

Pregunta 5. ¿Qué tipo de molde utilizo para el ladrillo?

Respuestas

Horno 1: alto $8 \mathrm{~cm}$; ancho $15 \mathrm{~cm}$; largo $28,5 \mathrm{~cm}$

Horno 2: alto $8 \mathrm{~cm}$; ancho $15 \mathrm{~cm}$; largo $28,5 \mathrm{~cm}$

Horno 3: alto $8 \mathrm{~cm}$; ancho $15 \mathrm{~cm}$; largo $28 \mathrm{~cm}$ 
Horno 4: alto $8 \mathrm{~cm}$; ancho $15 \mathrm{~cm}$; largo $28 \mathrm{~cm}$

Horno 5: alto $8 \mathrm{~cm}$; ancho $15 \mathrm{~cm}$; largo $28,5 \mathrm{~cm}$

Horno 6: alto $8 \mathrm{~cm}$; ancho $15 \mathrm{~cm}$; largo $28,5 \mathrm{~cm}$

Pregunta 6. ¿Conoce usted cuánto pesa el ladrillo?

\section{Respuestas}

Horno 1: los ladrillos tienen un peso de $5 \mathrm{~kg}$

Horno 2: no conoce, aproximadamente $4 \mathrm{~kg}$

Horno 3: no sabe

Horno 4: 4,5 kg depende, hay hornos en los que la arcilla pesa más y los ladrillos de $5 \mathrm{~kg}$

Horno 5: $5 \mathrm{~kg}$, aproximadamente

Horno 6: cada ladrillo pesa $5 \mathrm{~kg}$.

Pregunta 7. ¿Todos los ladrillos salen con la misma característica o varían? Respuestas

Horno 1: varían, mal quemados, original, se parten mucho, tamaño.

Horno 2: salen iguales
Horno 3: casi siempre de $28,5 \mathrm{~cm}$ de largo, $8 \mathrm{~cm}$ alto, $15 \mathrm{~cm}$ de ancho.

Horno 4: sí, la mayoría

Horno 5: lo ideal es que salgan iguales

Horno 6: lo mejor es que salgan iguales, debido a que es un proceso manual no salen todos iguales, la idea es disminuir el margen de error y que todos salgan casi con las mismas características.

Pregunta 8. ¿Usted cree que el peso del ladrillo es importante para su producto?

Respuestas

Horno 1: sí, porque, entre más pesa, más sufre el trabajador.

Horno 2: sí, para su mejor manejo

Horno 3: no tan importante, porque para venderlo no lo pesan.

Horno 4: sí, ya que los camiones tienen un límite para el peso.

Horno 5: es importante para ser transportado, son más fáciles los livianos.

Horno 6: aunque para venderlos no es pesado previamente, pero sí pienso que entre más liviano más fácil para nosotros poder moverlos. 
Pregunta 9. ¿Cuántos ladrillos se dañan por quema?

Respuestas

Horno 1: no conozco ese dato

Horno 2: no conozco ese dato

Horno 3: 40 ladrillos, aproximadamente

Horno 4: no conozco la cantidad

Horno 5: 50 ladrillos

Horno 6: más o menos 20 ladrillos

\section{Discusión de resultados}

No se observó ninguna diferencia en las técnicas de fabricación entre los hornos, todos siguen el mismo procedimiento, el mismo tipo de molde artesanal de madera, se infiere que la variación del peso promedio de los ladrillos de cada unidad no depende del método de fabricación, sino de otros factores como las características físico químicas de la materia prima, en este caso la arcilla.

Sin duda alguna, independientemente del horno y del sector en el que se ubica, sí existen variaciones en el peso de los ladrillos que se evidencian en los valores altos de desviación estándar y varianza.
Se hace notar que el horno seis produce ladrillos con menor desviación en el peso, lo que nos dice que son ladrillos mejores estandarizados, sin embargo, a primera vista no se aprecia diferencia de elaboración con las otras unidades, se infiere que es debido a la habilidad y experiencia que presenta el trabajador, ya que el horno es de mayor capacidad y la producción es mayor en comparación con los otros hornos.

En cuanto a la apreciación de los ladrilleros, la encuesta realizada establece lo siguiente:

- El peso no es tenido en cuenta para la comercialización del producto en la ladrillera, solo se tienen en cuenta las dimensiones.

- De los seis hornos encuestados, cuatro nos dicen que la importancia del peso del ladrillo radica en la facilidad para acarrearlo y transportarlo.

- Existe la creencia entre ellos que el peso del ladrillo depende del "peso de la arcilla", aspecto que entendemos como la posible diferencia de la densidad del material arcilla entre un sector y otro.

Una vez obtuvimos los datos de la compresión concluimos que el peso de los ladrillos no influye directamente con la resistencia de los ladrillos, deduci- 
mos que la resistencia de las unidades se ve afectada por otros factores, como la compactación de la mezcla y alguna alteración la elaboración normal de los ladrillos.

\section{Conclusiones}

Para concluir, las técnicas que utilizan los alfareros en el proceso de elaboración del ladrillo son las mismas en cada uno de los hornos, todos siguen el mismo procedimiento, el mismo tipo de molde artesanal de madera, se infiere que la variación del peso promedio de los ladrillos de cada unidad depende de las características físicas y químicas de la arcilla.

Se evidencian variaciones en el peso de los ladrillos en los valores altos de desviación estándar y varianza.

Se hace notar que el horno seis produce ladrillos con menor desviación en el peso, lo que nos dice que son ladrillos mejores estandarizados, sin embargo, a primera vista no se aprecia diferencia de elaboración con las otras unidades, se infiere que es debido a la habilidad y experiencia que presenta el trabajador, ya que el horno es de mayor capacidad y la producción es mayor en comparación con los otros hornos.

De la opinión de los trabajadores se concluye que el peso del ladrillo no es tenido en cuenta para la comercialización del producto en la ladrillera, solo se tienen en cuenta las dimensiones, de los seis hornos encuestados, cuatro nos dicen que la importancia del peso del ladrillo radica en la facilidad para acarrearlo y transportarlo. Existe la creencia entre ellos que el peso del ladrillo depende del "peso de la arcilla", aspecto que entendemos como la posible diferencia de la densidad del material arcilla entre un sector y otro.

Se propone a la ladrillera tener parámetros claros de control para la elaboración de los ladrillos, es decir, estandarizar el proceso, para que todo el producto salga con la misma calidad.

Una vez obtuvimos los datos de la compresión concluimos que el peso de los ladrillos no influye directamente con la resistencia de los ladrillos, deducimos que la resistencia de las unidades se ve afectada por otros factores, como la compactación de la mezcla y alguna alteración la elaboración normal de los ladrillos. 


\section{Referencias}

Corral, Y. (2010). Diseño de cuestionarios para recolección de datos. Revista Ciencias de la Educación, 20(36).

JAC de Las Casitas. (2006). Informe final de exploración y plan de trabajo y obras. Contrato de concesión 0175-20. Documento interno, Valledupar, Colombia.

Gonzáles, F. (2008). Proceso de fabricación del ladrillo. Puebla, México: Universidad Politécnica de Puebla, Ingeniería Electrónica y Telecomunicaciones.

Norma Técnica Colombiana (NTC 4205). Primera actualización, 2011. Bogotá, D. C.

Norma Colombiana de Diseño y Construcción Sismo Resistente NSR98. 